\title{
REGULAR PURE PROJECTIVE MODULES
}

\author{
DAVID J. FIELDHOUSE
}

\begin{abstract}
A structure theorem for regular pure projective modules over any associative ring with identity is given. This extends an earlier result for regular projective modules, which in turn extended a theorem of Kaplansky on the structure of projective modules over regular rings.
\end{abstract}

1. Introduction. Throughout this paper the word ring will mean an associative but not necessarily commutative ring with identity; all modules are unital.

In [2] the author proved a structure theorem for regular projective modules over any ring, which generalized a theorem of Kaplansky [3] giving the structure of projective modules over regular rings. Here we extend these results to a much wider class of modules: the regular pure projectives.

2. Purity and finitely presented modules. If $P \subseteq M$ are left $A$-modules, Cohn [1] calls $P$ pure in $M$ iff $X \otimes P \rightarrow X \otimes M$ is monic for all right $A$ modules $X$. We have called a module regular iff every submodule is pure. Basic properties of purity and regularity have been studied by the author in [2]. A ring is von Neumann regular iff every module over it is regular; hence theorems about regular modules generalize those about modules over regular rings.

A module $G$ is finitely presented iff there exists a short exact sequence $0 \rightarrow E \rightarrow F \rightarrow G \rightarrow 0$ with both $E$ and $F$ finitely generated and $F$ free. We will need the following

Proposition 2.1. A module $Z$ is finitely presented iff there exists $a$ short exact sequence $0 \rightarrow X \rightarrow Y \rightarrow Z \rightarrow 0$ with $X$ finitely generated and $Y$ finitely presented.

Received by the editors July $28,1971$.

AMS 1970 subject classifications. Primary 16A50, 16A30; Secondary 16A62, 16A64. Key words and phrases. Projective, pure projective, regular.

(c) American Mathematical Society 1972 
Proof. $\quad(\Rightarrow)$ Trivial.

$(\Leftarrow)$ There exists a commutative diagram, with exact rows and columns:

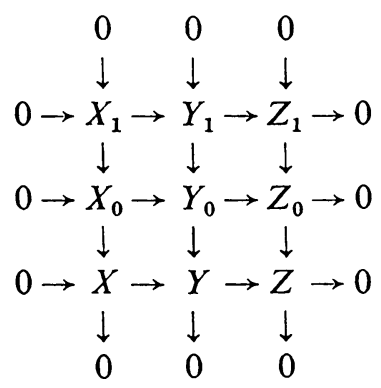

in which $Y_{0}=X_{0} \oplus Z_{0}, X_{0}$ and $Z_{0}$ are finitely generated free modules (and hence $Y_{0}$ too). Since $Y$ is finitely presented $Y_{1}$ is finitely generated and hence so is $Z_{1}$, i.e., $Z$ is finitely presented.

A module is pure projective iff it is projective for the pure exact sequences; pure projectives are just direct summands of direct sums of finitely presented modules. (See [2].) In particular projectives are pure projective.

3. Relative coretractions. The results in this section will be stated for the category of left $A$-modules, but in many cases could be extended to more general categories.

If $\alpha$ and $\varphi$ are maps such that $\alpha \varphi$ is defined, we say that $\alpha$ has a (relative) coretraction w.r.t. $\varphi$ iff there exists $\alpha^{\prime}$ with $\alpha^{\prime} \alpha \varphi=\varphi$, in which case we write $(\alpha, \varphi)$. Clearly $(\alpha, 1)$ iff $(\alpha, \varphi)$ for all $\varphi$ (for which $\alpha \varphi$ is defined, of course); in this case $\alpha$ has a coretraction. Note that if $\varphi=\varphi_{m} \varphi_{e}$ with $\varphi_{m}$ monic and $\varphi_{e}$ epic then $(\alpha, \varphi)$ iff $\left(\alpha, \varphi_{m}\right)$.

Proposition 3.1. Consider the following conditions:

(1) $(\alpha, \varphi)$ and $(\beta, \alpha \varphi)$;

(2) $(\beta \alpha, \varphi)$;

(3) $(\alpha, \varphi)$.

Then $(1) \Rightarrow(2) \Rightarrow(3)$. Hence if $(\beta, 1)$ we have $(\alpha, \varphi)$ iff $(\beta \alpha, \varphi)$.

Proof. Obvious.

THEOREM 3.2. If $\left(\alpha_{i}, \varphi_{i}\right)$ where $\left\{\alpha_{i}\right\}$ and $\left\{\varphi_{i}\right\}$ are directed systems over a directed set I then $(\alpha, \varphi)$ where $\alpha=\operatorname{inj} \lim \alpha_{i}$ and $\varphi=\operatorname{inj} \lim \varphi_{i}$.

Proof. Easy.

We will say that $(\alpha, \varphi)$ holds for all finitely generated $\varphi$ iff $(\alpha, \varphi)$ holds whenever the domain of $\varphi$ is finitely generated. 
THEOREM 3.3. If $\alpha: P \rightarrow M$ is monic and $(\alpha, \varphi)$ holds for all finitely generated $\varphi$ then $\alpha$ is pure (i.e. $P$ is pure in $M$ ). The converse holds if $M$ is pure projective.

Proof. Suppose we have a test situation (see [2]): i.e. a commutative diagram:

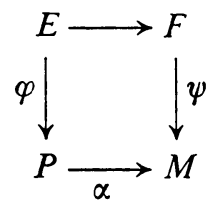

where both $E$ and $F$ are finitely generated free modules.

Let $K=\varphi E$, which is finitely generated. Since $(\alpha, \varphi)$ holds we have $\alpha^{\prime} \alpha \varphi=\varphi$ for some $\alpha^{\prime}: M \rightarrow P$. Define $\sigma=\alpha^{\prime} \psi$ which makes the upper left hand triangle commute, i.e. $P$ is pure in $M$.

Conversely, assume $M$ is pure projective and $\varphi$ is finitely generated, i.e. $\varphi: K \rightarrow P$ with $K$ finitely generated. Our earlier remarks show that without loss of generality we may assume that $\varphi$ is monic, i.e. $K \subseteq P \subseteq M$, and that $M=\oplus_{I} M_{i}$ with each $M_{i}$ finitely presented. Since $K$ is finitely generated, $K \subseteq M^{\prime}=\oplus_{I^{\prime}} M_{i}$ where $I^{\prime}$ is a finite subset of $I$, and hence $M^{\prime}$ is finitely presented.

Hence we have a commutative diagram (the test situation again):

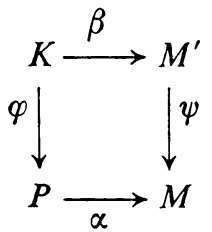

Since $M^{\prime} / K$ is finitely presented and $P$ is pure in $M$, there exists $\sigma: M^{\prime} \rightarrow P$ such that $\sigma \beta=\varphi$. Let $\pi: M \rightarrow M^{\prime}$ be the natural projection and define $\alpha^{\prime}=\sigma \pi$. Then $\alpha^{\prime} \alpha \varphi=\sigma \pi \alpha \varphi=\sigma \pi \psi \beta=\sigma \beta=\varphi$, and $(\alpha, \varphi)$ holds.

COROllary. If $P$ is pure in a pure projective module $M$, then for every finitely generated submodule $K$ of $P$ there exists a relative coretraction $\theta_{K}: M \rightarrow P$ with $\theta_{K}$ the identity on $K$. Hence a finitely generated submodule of a pure projective module is pure iff it is a direct summand.

We will call a module $M$ finitely pure coretractable iff whenever $K \subseteq P \subseteq M$ holds with $K$ finitely generated and $P$ pure in $M$ then there exists a relative coretraction of $\alpha: P \rightarrow M$ w.r.t. $\varphi: K \rightarrow P$. By the preceding result, every pure projective module is finitely pure coretractable. 
4. The structure theorems. We first prove

THEOREM 4.1. A module $M$ is the countable direct sum of cyclic modules iff it has a countable set of generators with the property that for each integer $n \geqq 1$ the submodule generated by the first $n$ generators is a direct summand.

Proof. $\quad(\Rightarrow)$ Obvious.

(«) Suppose $M$ has generators $x_{1}, x_{2}, \cdots$, and write $M_{n}=\sum_{1}^{n} A x_{i}$ for each integer $n \geqq 1$. Define inductively elements $y_{1}, y_{2}, \cdots$ in $M$ such that $M_{n}=\oplus_{1}^{n} A y_{i}$ for all $n$ and $M=\oplus_{1}^{\infty} A y_{i}$ as follows: let $y_{1}=x_{1}$ and assume $y_{i}$ defined for all $i \leqq n$. Decompose $x_{n+1}$ w.r.t. $M=M_{n} \oplus M_{n}^{\prime}$ as $x_{n+1}=z_{n+1}+y_{n+1}$ to define $y_{n+1}$. Clearly $\sum_{1}^{n+1} A x_{i}=M_{n+1}=M_{n} \oplus A y_{n+1}$ and $M=\oplus_{1}^{\infty} A y_{i}$.

COROLLARY. Every countably generated regular finitely pure coretractable module is the countable direct sum of regular cyclic modules.

THEOREM 4.2. A module is regular and pure projective iff it is the direct sum of finitely presented cyclic regular modules. If the module is regular and projective, the components of the direct sum are isomorphic to one-sided ideals which are direct summands of the ring.

Proof. $\quad(\Leftrightarrow)$ Direct sums of regular modules are regular. See [2].

$(\Rightarrow)$ Since every pure projective is the direct sum of countably generated pure projectives [2] we may apply the preceding results and observe that every finitely generated pure projective is finitely presented [2]. The projective case is immediate.

\section{REFERENCES}

1. P. M. Cohn, On the free product of associative rings, Math. Z. 71 (1959), 380-398. MR 21 \#5648.

2. D. J. Fieldhouse, Pure theories, Math. Ann. 184 (1969), 1-18. MR 40 \#5699.

3. I. Kaplansky, Projective modules, Ann. of Math. (2) 68 (1958), 372-377. MR 20 \#6453.

Department of Mathematics, University of Guelph, Guelph, Ontario, Canada 